\title{
Nonalcoholic Fatty Liver Disease and Chronic Vascular Complications of Diabetes
}

Giovanni Targher ${ }^{1}$, Amedeo Lonardo ${ }^{2}$, Christopher D. Byrne ${ }^{3,4}$

${ }^{1}$ Department of Medicine, Section of Endocrinology, Diabetes and Metabolism, University and Azienda Ospedaliera Universitaria Integrata of Verona, Piazzale Stefani 1, 37126 Verona, Italy

${ }^{2}$ Department of Internal Medicine and Metabolic Diseases, Nuovo Ospedale Sant'Agostino Estense di Baggiovara, Via Giardini 1355, 41126 Baggiovara, Modena, Italy

${ }^{3}$ Nutrition and Metabolism, Faculty of Medicine, University of Southampton, UK

${ }^{4}$ Southampton National Institute for Health Research Biomedical Research Centre, University Hospital Southampton, Southampton General Hospital, Tremona Road, Southampton SO16 6YD, UK

Word count: Abstract: 197 words; Text: 6667 (excluding title page, abstract, references, figure legends, and key points); Table: 1 + supplementary Tables: 2; Figures: 6; References: 185

Address for correspondence:

Prof. Giovanni Targher, M.D.

Section of Endocrinology, Diabetes and Metabolism

Department of Medicine

University and Azienda Ospedaliera Universitaria Integrata of Verona

Piazzale A. Stefani, 1

37126 Verona, Italy

Phone: +39-0458123110

E-mail: giovanni.targher@univr.it 


\section{List of abbreviations used}

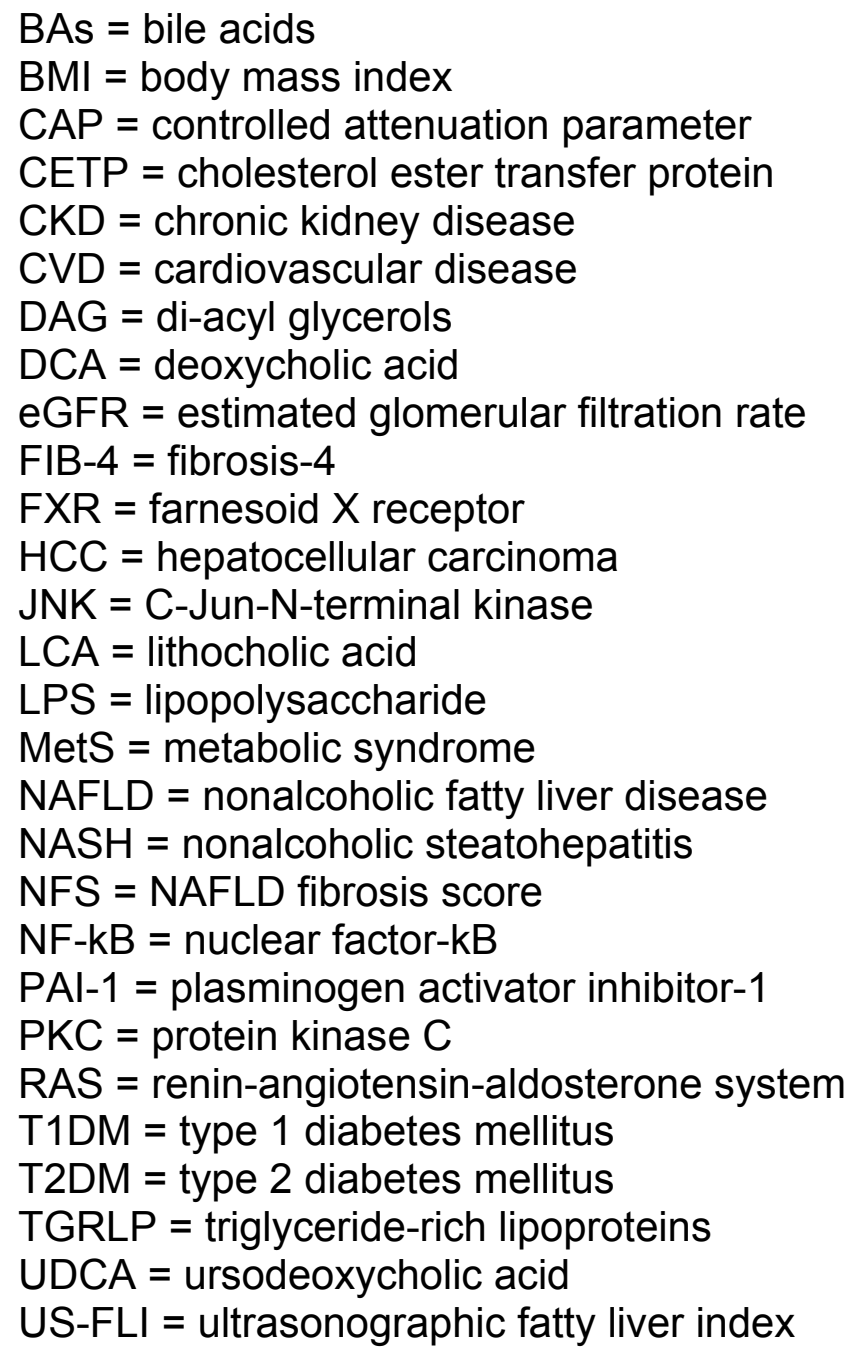




\section{ABSTRACT}

Nonalcoholic fatty liver disease (NAFLD) and diabetes mellitus are common diseases that often coexist and may act synergistically to increase risk of hepatic and extrahepatic clinical outcomes. NAFLD affects up to 70-80\% of patients with type 2 diabetes and up to $30-40 \%$ of adults with type 1 diabetes. The coexistence of NAFLD and diabetes increases not only the risk of developing the more severe forms of NAFLD but also the risk of developing chronic vascular complications of diabetes. Indeed, substantial evidence links NAFLD with risk of developing cardiovascular disease and other cardiac and arrhythmic complications in patients with type 1 or type 2 diabetes. NAFLD is also associated with risk of developing microvascular diabetic complications, especially chronic kidney disease. The review focuses on the strong association between NAFLD and risk of chronic vascular complications both in patients with type 1 and type 2 diabetes, thereby promoting an increased awareness of the extra-hepatic implications of this increasingly prevalent liver disease. We also discuss the putative underlying mechanisms by which NAFLD contributes to vascular diseases, and includes a discussion of the emerging role of changes in the gut microbiota (dysbiosis) in the pathogenesis of NAFLD and associated vascular diseases. 


\section{INTRODUCTION}

Nonalcoholic fatty liver disease (NAFLD) describes, in the absence of competing etiologies of liver injury, a variable combination of individual histological elementary findings, namely accumulation of fat in more than $5 \%$ of hepatocytes, often with minor degrees of low-grade sterile inflammation (simple steatosis); steatosis plus ballooning degeneration (nonalcoholic steatohepatitis [NASH]), advanced fibrosis and "cryptogenic" cirrhosis ${ }^{1,2}$.

NAFLD has become among the most common chronic liver diseases in many parts of the world; it occurs in up to $30 \%$ of adults in the general population in Western countries and the prevalence of the disease is even much greater in patients with type 2 diabetes mellitus (T2DM), who are also more likely to develop the more severe histological forms of NAFLD [i.e., NASH, advanced fibrosis, cirrhosis and hepatocellular carcinoma $(\mathrm{HCC})]^{1-3}$.

Strong evidence now indicates that the global health burden of NAFLD is not only confined to severe liver-related complications (cirrhosis, liver failure, HCC and liver transplantation) but also embraces major extra-hepatic conditions ${ }^{4-6}$. Indeed, the leading causes of mortality among patients with NAFLD are cardiovascular disease (CVD), followed by non-liver malignancy and liver disease ${ }^{1-3}$. Moreover, as it will be discussed in greater detail below, it has also become increasingly clear that the presence and severity of NAFLD is strongly associated with an increased risk of developing serious extra-hepatic diseases, such as, further to CVD, cardiomyopathy and cardiac arrhythmias as well as chronic kidney disease (CKD), which ranks among the most important chronic complications of diabetes.

The present review focuses on the adverse impact of NAFLD on the risk of chronic vascular complications of diabetes (principally CVD, CKD but also other microvascular diabetic complications). In addition, the putative pathophysiological mechanisms by which NAFLD may contribute to the development and progression of chronic vascular complications of diabetes are also discussed. Finally, the principles of NAFLD treatment are also critically evaluated.

\section{DIAGNOSIS AND EPIDEMIOLOGY OF NAFLD}




\subsection{Diagnostic methods}

NAFLD remains a diagnosis "of exclusion", which suffers from the contradiction of missing a positive diagnostic criterion useful to define $i^{7,8}$. This implies that in operational terms ${ }^{1,9,10}$ and irrespective of the background population, the clinicians will have: a) to identify excess hepatic fat content by using various imaging technologies or, in some cases, by liver biopsy, which remains the "reference standard" for diagnosing $\mathrm{NASH}$ and staging the severity of necroinflammation and fibrosis in patients with NAFLD; b) to exclude alcoholic, viral, pharmacological, autoimmune and inheritedgenetic etiologies of steatotic liver disease; c) to ascertain the coexistence of the typical features of metabolic syndrome $(\mathrm{MetS})^{11}$, and d) to assess the severity of hepatic fibrosis, which is the strongest predictor for disease-specific mortality in NAFLD ${ }^{1-3,6}$. Clearly, point c) may be superfluous when dealing with people with diabetes.

\subsection{Possible screening strategies}

Screening for NAFLD among patients with established diabetes should be a multistep process.

The presence of fat vesicles in at least $5 \%$ of hepatocytes defines steatosis histologically ${ }^{1,2}$. However, since liver biopsy is an invasive procedure that cannot be proposed for all patients with suspected NAFLD, both non-invasive biomarkers of hepatic steatosis and imaging techniques have been developed. To date, non-invasive biomarkers of steatosis have a limited clinical utility, as they often do not accurately quantify hepatic steatosis as assessed histologically ${ }^{12}$. Therefore, imaging techniques are the preferred diagnostic tests for assessing liver fat accumulation. Proton magnetic resonance spectroscopy is the most precise method for measuring hepatic triglyceride content, but it is of limited availability owing to its high costs ${ }^{1,2}$. Ultrasonography is the most widely used imaging method in clinical practice, and has a diagnostic accuracy of 0.91-0.93 for detecting mild-to-moderate hepatic steatosis and a specificity of 0.88$0.99^{13}$. The accuracy of ultrasonography may further improve in relation to the local expertise and the availability of newer ultrasound machines ${ }^{14}$. Semi-quantitative ultrasonographic indices may also provide added diagnostic value ${ }^{14}$. For example, the ultrasonographic fatty liver index (US-FLI) with a cut-off value $\geq 2$ detects a minimum amount of $10 \%$ steatosis on liver histology (sensitivity $90 \%$, specificity $90 \%)^{15}$. Moreover, a US-FLI $<4$ has also a $94 \%$ negative predictive value for excluding severe 
$\mathrm{NASH}^{16}$. Controlled attenuation parameter (CAP) assessed by transient elastography (FibroScan) at a cut-off value of $310 \mathrm{~dB} / \mathrm{m}$ has $80 \%$ sensitivity, $71 \%$ specificity, $86 \%$ positive and $71 \%$ negative predictive values, respectively, for histological steatosis $>30 \%{ }^{17}$.

The exclusion of competing etiologies of chronic liver disease can be accomplished through medical history, simple biochemical parameters ${ }^{1,2,9}$ and specific questionnaires to exclude excessive alcohol consumption ${ }^{18}$.

The ascertainment of a full-blown MetS will be accomplished, at baseline and repeated over time, through appropriate family and personal history; physical examination and a first-level laboratory assessment ${ }^{1,9}$. Interestingly, the US-FLI may better differentiate the presence of MetS than insulin resistance alone ${ }^{15,16,19}$.

Staging of hepatic fibrosis can be implemented with the use of multiple non-invasive methods. These include both "biochemical" score systems [e.g., fibrosis-4 score (FIB-4) $>2.67$, NAFLD fibrosis score (NFS) $>0.676$ and enhanced liver fibrosis score (ELF) $>10.51$ ] and "physical" techniques (e.g., liver stiffness measurement [LSM] assessed with transient elastography $>9.6 \mathrm{KPa}$ or with other non-invasive imaging methods) ${ }^{20,21}$. The serial combination of LSM with FIB-4/NFS measurements accurately predicts the presence of advanced hepatic fibrosis in NAFLD ${ }^{21}$.

In the schematic Figure 1 we propose a pragmatic algorithm for the diagnosis and monitoring of NAFLD in patients with established diabetes. However, it is important to emphasize that an intense debate on aspects of our as well as of similar algorithms is ongoing, and that a validated, widely accepted, algorithm for the diagnosis and monitoring of NAFLD in patients with established diabetes does not yet exist. In particular, screening for NAFLD (both in the general population and in high-risk patient groups) is not universally recommended by all scientific societies ${ }^{1,2,22,23}$; that its costeffectiveness remains controversial ${ }^{24,25}$ and that liver biopsy should be eventually carried out in at least a (large) proportion of those submitted to non-invasive screening, given that the diagnosis of $\mathrm{NASH}$ remains universally based on histological grounds ${ }^{1,2,19,21,22}$. For example, a recent cross-sectional study conducted in approximately 122,000 patients with T2DM found a high prevalence of advanced 
hepatic fibrosis using NFS and other non-invasive scores ${ }^{26}$; however, the significant variability among the findings provided by such scores (ranging from nearly $9 \%$ with the use of the FIB-4 score to nearly $35 \%$ with NFS) strongly supports the need for their further validation in diabetic populations ${ }^{26}$.

\subsection{Epidemiology}

Around one-quarter of adults in the United States and Europe have NAFLD, and in certain areas of South America and Asia the prevalence of the disease is even higher ${ }^{27}$. This finding further highlights the overwhelming potential clinical and economic burdens imposed by NAFLD, which is now projected to increase further in the foreseeable future $^{1,27}$. The scale of the burden of NAFLD also implies that no Health Authority worldwide can afford to promote any screening campaigns aimed at identifying NAFLD in the general population.

\subsubsection{T2DM}

On these grounds, the identification of certain selected cohorts of individuals at high risk of developing NAFLD (such as people with T2DM) appears to be a more fruitful strategy ${ }^{1,2,11}$ Irrespective of the characteristics of the cohorts studied (hospital-based vs. population-based cohorts) and the diagnostic methodologies used for diagnosing NAFLD (imaging vs. biopsy), the prevalence of the disease has been found to be much greater in patients with T2DM than in the nondiabetic population, ranging from nearly $40 \%$ to $100 \%$ (as summarized in the Supplementary Table 1$)^{28-44}$.

Patients with T2DM are also more likely to have more severe histological forms of NAFLD, such as NASH with advanced fibrosis, even with fairly normal serum aminotransferase levels ${ }^{33,35}$. Therefore, serum aminotransferase levels are not reliable indicators for the screening and diagnosis of NAFLD among patients with T2DM, and should not be used to this end in clinical practice ${ }^{1-4,9,45}$.

Notably, the coexistence of NAFLD and T2DM will worsen the course of both diseases $^{45-49}$. Coexisting T2DM not only increases the risk of NAFLD progression to advanced fibrosis and cirrhosis, but also increases the risk of incident HCC, liver-related hospital admissions and liver-related deaths ${ }^{43,49-55}$. Conversely, the presence of NAFLD makes achieving good glycaemic control more difficult, increases hepatic and peripheral 
insulin resistance and exacerbates atherogenic dyslipidaemia ${ }^{1-3,45,47}$; thus further increasing the risk of incident $\mathrm{CKD}^{4-6,56}$ and major CVD events, particularly in those patients with advanced NAFLD ${ }^{1,57,58}$.

Collectively, these findings strongly support the assertion that in patients with T2DM, diagnosis of, and treatment for, NAFLD should be considered a high priority for diabetologists or endocrinologists caring for patients at risk of NAFLD.

\subsubsection{T1DM}

Compared to the heavy toll imposed on the liver by T2DM, epidemiological data on the burden of NAFLD imposed by T1DM (i.e., a disease that is characterized by an altered porto-systemic gradient of insulin and a lesser degree of insulin resistance compared to T2DM) appear to be more variable (summarized in Supplementary Table 2) ${ }^{59-67}$.

Some authors have reported a (relatively) high prevalence of NAFLD (on ultrasonography), up to nearly $50 \%$, in adult patients with $\mathrm{T}_{1 \mathrm{DM}} \mathrm{D}^{60,61}$. Others have reported a prevalence of NAFLD (diagnosed by magnetic resonance imaging) of $30 \%$ in a small group of adults with $\mathrm{T}_{1} \mathrm{DM}^{67}$. However, some investigators have disputed this notion by reporting a prevalence of NAFLD (on magnetic resonance imaging) in patients with T1DM that ranged from $0 \%$ (in children with T1DM) ${ }^{63}$ to nearly $10-15 \%{ }^{65,66}$, which is a prevalence of the disease definitely lower than that observed in the general adult population.

We consider that these wide inter-study differences in the prevalence of NAFLD might be, at least in part, due to differences in the imaging techniques used to diagnose NAFLD as well as to differences in age, sex distribution, duration of diabetes, family history of T2DM, body mass index (BMI) and degree of glycaemic control among the various cohorts of T1DM patients studied. A large, prospective UK study of adult patients with T1DM and T2DM, who had undergone liver biopsy, has reported that those with T1DM had a risk of developing cirrhosis and portal hypertension, which was comparable to that observed in T2DM individuals who were matched for sex, age, diabetes duration, obesity and other potential confounding variables ${ }^{68}$. However, we suggest that further larger studies of well-characterized T1DM patients are certainly required to better characterize the relationship between NAFLD and T1DM. 


\section{STUDIES LINKING NAFLD TO RISK OF CHRONIC VASCULAR COMPLICATIONS OF DIABETES}

In recent years, many epidemiological studies have documented that NAFLD, diagnosed either by imaging or by histology, is associated with a substantially increased risk of all-cause and cause-specific (cardiovascular, cancer-related and liver-related) mortality both in nondiabetic patients and in those with $\mathrm{T}_{2} \mathrm{DM}^{1-3}$. Strong evidence indicates that CVD is a clinical concern in NAFLD, and that patients with NAFLD are more likely to experience CVD-related death than liver-related death ${ }^{4-6,69}$. Furthermore, several studies have also suggested that NAFLD is associated with an increased risk of chronic vascular complications of diabetes ${ }^{47}$.

\subsection{Macrovascular complications}

Substantial epidemiological evidence links NAFLD with various markers of subclinical atherosclerosis (e.g., increased arterial stiffness, endothelial dysfunction or increased carotid and lower limb atherosclerotic plaques) and with an increased prevalence of clinically manifest CVD across different patient populations, including people with $\mathrm{T}_{2} \mathrm{DM}^{3.4,58,69,70}$. Recently, Guo et al. confirmed that ultrasound-diagnosed NAFLD was associated with an increased prevalence of carotid and lower limb atherosclerotic plaques, independent of conventional CVD risk factors, duration of diabetes, haemoglobin A1c, insulin resistance, serum liver enzyme levels and medication use in a large cohort of Chinese individuals with $\mathrm{T}_{2} \mathrm{DM}^{71}$. Again, the Valpolicella Diabetes Heart Study, including 2392 Italian outpatients with T2DM without secondary causes of chronic liver disease, demonstrated that those with ultrasound-diagnosed NAFLD had a remarkably greater prevalence of clinically manifest coronary, cerebrovascular and peripheral vascular disease (Figure 2), independent of age, sex, BMI, waist circumference, smoking, low-density lipoprotein (LDL)-cholesterol, haemoglobin A1c, duration of diabetes, presence of MetS and use of hypoglycaemic, antihypertensive, lipid-lowering and antiplatelet medications ${ }^{28}$. Almost similar results were observed in smaller cohorts of adult patients with T1DM, where NAFLD (diagnosed by ultrasonography) was associated with higher odds of prevalent CVD, independently of age, sex, BMI, smoking, duration of diabetes, haemoglobin A1c, systolic blood pressure, plasma lipids and use of medications ${ }^{60,61}$. Moreover, in both nondiabetic and diabetic patients referred for clinically indicated coronary angiography, NAFLD was 
associated with a greater severity of coronary artery disease and with an increased prevalence of high-risk and vulnerable coronary artery plaques, independently of the extent and severity of coronary atherosclerosis ${ }^{70,72-74}$.

To date, convincing evidence also substantiates the existence of a strong link of NAFLD with subclinical myocardial remodeling and dysfunction (i.e., left ventricular diastolic dysfunction and cardiac hypertrophy), valvular heart diseases (i.e., aortic-valve sclerosis and mitral annulus calcification) and cardiac arrhythmias (mainly atrial fibrillation and QTC interval prolongation on standard electrocardiograms) both in patients with and without diabetes ${ }^{75-84}$. Preliminary evidence also suggests that ultrasound-diagnosed NAFLD, irrespective of pre-existing diabetes, is associated with an increased risk of 1-year all-cause and cardiac re-hospitalizations in patients admitted with acute heart failure ${ }^{85}$.

Available data leave little doubt that NAFLD is consistently associated with an increased prevalence of CVD and other cardiac and arrhythmic complications across a wide range of patient populations, including those with diabetes. However, whether NAFLD is an independent CVD risk factor or simply a bystander that shares common aetiological factors remains still debatable.

To date, a number of large hospital-based and population-based cohort studies reported an increased incidence of fatal and nonfatal CVD events in patients with either imaging- or biopsy-diagnosed NAFLD, independent of conventional CVD risk factors, both in patients with and without diabetes ${ }^{1,4-6,58,69,70}$. For instance, a prospective nested case-control study in 744 Italian outpatients with T2DM, who were free of diagnosed CVD at baseline, demonstrated that those with ultrasound-diagnosed NAFLD had a nearly twofold increased risk of developing nonfatal coronary heart disease, ischemic stroke or cardiovascular death over a follow-up period of 5 years. Notably, this association was independent of age, sex, smoking, diabetes duration, haemoglobin A1c, LDL-cholesterol, serum liver enzymes, presence of MetS, and use of hypoglycaemic, antihypertensive, lipid-lowering and antiplatelet medications ${ }^{86}$. Almost identical results were confirmed in a subsequent study with a larger sample size $(n=2103)$ and a longer follow-up period (6.5 years) ${ }^{87}$. Similarly, in a cohort of 286 adult outpatients with T1DM, the presence of ultrasound-diagnosed NAFLD was associated 
with a nearly six-fold increased risk of nonfatal CVD events (i.e., combined endpoint inclusive of nonfatal ischaemic heart disease, ischaemic stroke or coronary/peripheral revascularization procedures) over a mean follow-up period of 5.3 years ${ }^{88}$. Notably, this association was independent of age, sex, BMI, smoking, diabetes duration, haemoglobin A1c, dyslipidaemia, hypertension, CKD, prior ischaemic heart disease and serum gamma-glutamyltransferase levels ${ }^{88}$.

Recently, an updated and large meta-analysis that incorporated almost 34000 individuals with and without T2DM (36.3\% with NAFLD) and approximately 2600 fatal and nonfatal CVD outcomes ( $>70 \%$ CVD deaths) in 16 unique, observational prospective and retrospective cohort studies from different countries concluded that the presence of NAFLD (as detected by either imaging or histology) was associated with a higher risk of incident fatal and nonfatal CVD events over a median follow-up period of 6.9 years (random-effect odds ratio $1.64,95 \% \mathrm{Cl} 1.3-2.1$ ), and that this risk increased further with greater severity of NAFLD (random-effect odds ratio $2.58 ; 95 \% \mathrm{Cl} 1.8-3.8)^{57}$. Sensitivity analyses did not alter these findings. In particular, limiting the analysis to high-quality studies ( $n=10$ studies; random-effect odds ratio $1.54,95 \% \mathrm{Cl} 1.1-2.1$ ) and limiting to studies with full adjustment for covariates ( $n=5$ studies; random-effect odds ratio $1.69,95 \% \mathrm{Cl} 1.3-2.6)$ provided overall risk estimates consistent with the primary analysis $^{57}$.

Although further research is needed to definitely establish a causal association between NAFLD and increased risk of incident CVD events, the current evidence from the published studies (which have been performed among various ethnic populations with different lifestyle habits) supports the notion that a diagnosis of NAFLD identifies a subset of patients, who are at greater risk of CVD mortality and morbidity over time. In line with this assertion, the recent European clinical practice guidelines for the diagnosis and management of NAFLD have strongly recommended CVD risk assessment in all patients with NAFLD ${ }^{1}$.

\subsection{Microvascular complications}

NAFLD is significantly associated with the risk of microvascular diabetic complications, especially with CKD. In particular, numerous observational studies showed that NAFLD is associated with an increased prevalence of CKD (defined as estimated glomerular 
filtration rate $\left[\right.$ eGFR] $<60 \mathrm{ml} / \mathrm{min} / 1.73 \mathrm{~m}^{2}$ or proteinuria) both in patients without diabetes and in those with $\mathrm{T} 2 \mathrm{DM}$ or $\mathrm{T}_{1 \mathrm{DM}}{ }^{89}$. As shown in Figure 3, in the Valpolicella Heart Diabetes Study cohort involving 2103 Italian outpatients with T2DM without known chronic liver diseases and CVD at baseline, who had available measurements on eGFR, albuminuria and retinopathy ${ }^{90}$, it has reported that patients with ultrasounddiagnosed NAFLD had a nearly twofold increased risk of prevalent CKD or advanced (proliferative or laser-treated) diabetic retinopathy, independently of age, sex, BMI, waist circumference, smoking, hypertension, diabetes duration, haemoglobin A1c, plasma lipids and medication use. Conversely, in a subgroup of 5963 adult participants (15.8\% with established diabetes) of the National Health and Nutrition Examination Survey-III, the presence of ultrasound-diagnosed NAFLD was not significantly associated with any degree of retinopathy (detected via fundus photographs) both in individuals with and without known diabetes after adjusting for multiple covariates ${ }^{91}$. Some other studies in which NAFLD was diagnosed by either ultrasonography or histology have shown that the presence and severity of NAFLD was strongly associated with an increased prevalence of abnormal albuminuria or decreased kidney function in patients with T2DM or prediabetes ${ }^{89,92,93}$. Similarly, some smaller cohorts of Italian adult outpatients with T1DM have shown that ultrasound-diagnosed NAFLD was associated with the presence of diabetic retinopathy or nephropathy, independently of age, sex, BMI, hypertension, diabetes duration, haemoglobin A1c and use of medications ${ }^{94,95}$.

To date, there is a paucity of published data regarding the risk of developing CKD (or other microvascular complications) in patients with coexistent NAFLD and diabetes. In a subset of 1760 outpatients with T2DM of the Valpolicella Heart Diabetes Study, who had normal kidney function at baseline (i.e., after excluding those with CKD or macroalbuminuria [ $n=343]$ ), the presence of ultrasound-diagnosed NAFLD was associated with an increased risk of incident CKD (defined as occurrence of eGFR <60 $\mathrm{ml} / \mathrm{min} / 1.73 \mathrm{~m}^{2}$ or overt proteinuria) over a follow-up period of 6.5 years, independently of age, sex, smoking, BMI, waist circumference, diabetes duration, blood pressure, plasma lipids, haemoglobin A1c, baseline eGFR, albuminuria and use of hypoglycaemic, antihypertensive, lipid-lowering and antiplatelet drugs (adjusted-hazard ratio $1.49 ; 95 \% \mathrm{Cl} 1.1-2.2)^{96}$. Consistent with these findings, in a retrospective cohort study of 261 T1DM adults with preserved kidney function at baseline, who were followed-up for a mean period of 5.2 years, NAFLD (diagnosed by ultrasonography) 
was associated with an approximately threefold increased risk of incident CKD even after adjustment for age, sex, hypertension, diabetes duration, haemoglobin A1C and baseline eGFR ${ }^{97}$. Notably, addition of NAFLD to conventional cardio-renal risk factors significantly improved the discriminatory capability of the regression models for predicting $\mathrm{CKD}^{97}$.

A comprehensive meta-analysis of 33 observational, cross-sectional and prospective studies (including a total of 64000 individuals) confirmed that the presence and severity of NAFLD, as diagnosed by biochemistry, imaging or histology, was associated with a nearly twofold increase in the prevalence and incidence of CKD. In all of these analyses, the significant association between NAFLD and increased risk of CKD persisted after adjustment for pre-existing diabetes and other established cardio-renal risk factors ${ }^{98}$.

Finally, some studies also suggest that NAFLD is associated with an increased prevalence of distal symmetric polyneuropathy in patients with T1DM or T2DM ${ }^{99,100}$. Currently, however, published studies that have evaluated the existence of such association are very few and have produced conflicting results ${ }^{101,102}$.

Despite the growing evidence that links NAFLD with CKD and other microvascular complications in patients with T2DM or T1DM, a causal association remains to be definitely proven, and additional larger studies in different ethnic populations are needed to establish whether the steatotic/inflamed liver may actively contribute to the increased risk of microvascular complications observed among diabetic patients with NAFLD, a hypothesis which is biologically plausible, as discussed below.

\section{PUTATIVE BIOLOGICAL MECHANISMS BY WHICH NAFLD CONTRIBUTES TO THE DEVELOPMENT OF CHRONIC VASCULAR COMPLICATIONS OF DIABETES}

Several years ago, it was noted that T2DM and CVD share many risk factors (the "common soil" hypothesis ${ }^{103}$ ) and that unlike classical microvascular complications, large-vessel atherosclerosis can precede the development of T2DM. It has been recognized for many years that functioning of pancreatic $\beta$ cells, skeletal muscle, liver and adipose tissue are important in the development of T2DM and more recently 
functioning of other organs such as the intestine, brain and kidneys, as well as pancreatic $\alpha$ cells, has been highlighted in the development of chronic hyperglycaemia (the so-called "ominous octet") ${ }^{104}$. Thus, rather than vascular disease being a complication of diabetes, both conditions may have common antecedents, i.e., they spring from a "common soil" 103 and those common antecedents involve the functioning of other key organs beyond the pancreas.

As discussed previously, several authors have shown that NAFLD may be a novel risk factor for CVD, CKD and T2DM", and when taken in conjunction with the "common soil" hypothesis and the "ominous octet", it is now evident that NAFLD also shares many risk factors with diabetes and CVD. It is widely accepted that these shared risk factors revolve around ectopic fat accumulation (abdominal obesity), insulin resistance and other features of MetS ${ }^{105}$. Consequently, when considering the underlying mechanisms by which NAFLD may contribute to the development of chronic vascular complications of diabetes, it is important not only to consider the influence of the steatotic/inflamed liver per se, but also to consider the influence of abdominal obesity and other shared cardiometabolic risk factors. In particular, there is a cross-talk between the expanded/inflamed visceral adipose tissue and the liver, with this latter acting as both the target organ and the source of the systemic subclinical chronic inflammation and abnormalities in coagulation and fibrinolysis (as discussed below) that may promote an increased risk of developing chronic vascular complications of diabetes ${ }^{2-4,69,70}$.

Recently, there has also been considerable research interest in the possible pathogenic role of perturbations in the normal intestinal microflora (the so-called "dysbiosis") and abnormalities of normal intestinal function on CVD risk factors. In discussing the biological mechanisms underpinning the relationship between NAFLD and chronic vascular complications of diabetes, we will discuss the emerging evidence that suggests a link between dysbiosis, intestinal barrier dysfunction, gut microbialmediators and $C V D^{106-110}$. We will also discuss potential haemostatic, prothrombotic and proinflammatory mediators, as well as mechanisms contributing to oxidative stress that may link NAFLD to chronic vascular complications of diabetes.

\subsection{Consequences of dysbiosis}


Since the liver is the key metabolic organ exposed to high levels of intra-colonic fermentation products (via the portal vein), the changes in specific microbial products, secondary to altered gut microbial composition, and the changes in intestinal permeability and function can affect hepatic structure and function to further increase risk of NAFLD. Dysbiosis ${ }^{111}$ has recently been described in patients with MetS, and in those with established CVD ${ }^{107-109}$, T2DM ${ }^{112-115}$, NAFLD ${ }^{116-118}$ or CKD ${ }^{119}$. In Figure 4 are displayed the potential pathways, factors and processes that link dysbiosis/gut microbial mediators and NAFLD to CVD risk factors and vascular and renal diseases.

\subsubsection{Increased gut permeability and release of lipopolysaccharide into the circulation}

Dysbiosis is frequently associated with increased production of endotoxins from Gramnegative bacteria that can damage the intestinal barrier, affect nutrient harvesting and increase gut permeability with the potential for lipopolysaccharide (LPS) to enter the portal and systemic circulation to increase the inflammatory burden ${ }^{120,121}$. LPS causes disruption of the gut intracellular tight junctions, favouring the release of proinflammatory cytokines into the circulation and, consequently, into the liver ${ }^{120-123}$. Since LPS production provides a direct inflammatory stimulus to the liver via the portal vein, LPS might increase risk of hepatic inflammation and oxidative stress.

\subsubsection{Altered short-chain fatty acid production, trimethyl-amine metabolism, uraemic toxins and bile acid metabolism}

Fermentation of dietary fibre in the intestine by anaerobic bacteria, such as Lactobacilli and Bifidobacteria, forms short-chain fatty acids (SCFAs) ${ }^{124,125}$. SCFAs include acetate, propionate and butyrate that influence hepatic de novo lipogenesis, and gluconeogenesis. A meta-analysis of predominantly short-term probiotic treatments in T2DM has suggested a beneficial effect on insulin resistance, thought to be mediated by increasing butyrate production ${ }^{126}$. The current list of bacterially derived bioactive molecules that have the potential to adversely influence the vasculature includes: trimethylamine (TMA)/trimethylamine oxide (TMAO), secondary bile acids, LPS, and catecholamines $^{106-109}$. With dysbiosis there may also be an increase in uraemic toxins, such as TMAO, p-cresyl sulfate, 4-ethyl phenyl sulphate, hippuric acid, indoxyl sulfate and indole-3 acetic acid ${ }^{127}$. 
Increased TMA/TMAO levels have been shown to cause 'atherogenic' lesions in mice and are associated with atherosclerosis in $\operatorname{man}^{109}$, and it has been suggested that TMAO may exert a marked adverse effect on the vasculature, increasing carotid-artery intimal medial thickness ${ }^{128}$ to promote $C V D^{107}$. Experimentally, it has been shown that TMAO may impair reverse cholesterol transport, induce platelet aggregation, promote foam cell formation and increase expression of the scavenger receptors $A 1$ and CD36 ${ }^{109}$. TMAO is produced in the liver from the oxidation of TMA that is produced as a direct consequence of bacterially dependent metabolism of dietary choline.

\subsubsection{Altered bile acid metabolism}

Primary bile acids (BAs), such as cholic acid and chenodeoxycholic acid (CDCA), are produced by the liver. BAs interact with plasma membrane G-protein-coupled receptors (e.g., TGR5), muscarinic receptors and nuclear receptors, e.g. the farnesoid (FXR) and pregnane $(\mathrm{PXR}) \mathrm{X}$ receptors ${ }^{129}$. BA receptors are expressed in cardiovascular tissue, e.g. endothelial cells vascular smooth cells and cardiomyocytes ${ }^{129}$. CDCA is a naturally occurring ligand for the FXR, and activation of the FXR with modified CDCA compounds, such as obeticholic acid, is now known to have marked effects not only on bile acid metabolism but also on liver disease, cholesterol metabolism and LDLcholesterol levels in NAFLD ${ }^{130,131}$. Besides regulating bile acid metabolism, FXR activation also powerfully influences levels of lipids such as hepatic glucose output, hepatic glycogen, cholesterol, triglyceride and fatty acids, and also regulates inflammation. The BAs are influenced by gut microbiota to produce secondary bile acids, such as urodeoxycholic acid (UDCA), deoxycholic acid (DCA) and lithocholic acid (LCA). Secondary BAs are highly hydrophobic and toxic, and increased concentrations in the liver have been linked to inflammation ${ }^{132}$ and NAFLD ${ }^{133}$. There is also evidence suggesting that alteration of bile acid metabolism by the intestinal microbiota may influence CVD risk by affecting LDL-cholesterol metabolism, vasomotor tone and blood pressure $^{129,134}$. Furthermore, treatment with Bifidobacterium may influence cholesterol metabolism by decreasing serum total cholesterol and LDL-cholesterol concentrations $^{135}$. CDCA treatment has also recently been shown to decrease the LDLreceptor modulator proprotein convertase subtilisin/kexin type 9 (PCSK9) ${ }^{136}$, providing another potentially important mechanism by which BAs may modify cholesterol metabolism. Figure 5 summarizes potential pathways linking dysbiosis to vascular diseases. 


\subsection{Insulin resistance, atherogenic dyslipidaemia, proinflammatory cytokines, increased haemostatic factors and oxidative stress}

The development and, more importantly, progression of NAFLD result in amplifying the risk of vascular damage with an increase in other traditional and nontraditional CVD risk factors, such as atherogenic dyslipidaemia, insulin resistance, proinflammatory cytokines, haemostatic-fibrinolytic factors and increased oxidative stress.

The development of NAFLD is associated with the accumulation of intra-hepatic ceramides and di-acyl glycerols (DAG) ${ }^{4}$ as well as the secretion of multiple hepaticderived molecules, such as fetuin-A and other hepatokines that are able to inhibit the insulin receptor tyrosine kinase to promote hepatic and peripheral insulin resistance ${ }^{137}$. Indeed, intrahepatic accumulation of lipid intermediates, such as ceramides and DAG, inhibits insulin signaling. For example, DAG activates protein kinase C-epsilon (PKCepsilon) membrane translocation, inhibits the insulin receptor kinase and decreases insulin signaling; and increased hepatic ceramide results in activation of inflammatory toll-like receptor-4 signaling pathways, with consequent impairment of insulin signaling pathways via inhibition of Akt phosphorylation ${ }^{138,139}$.

The development of insulin resistance in NAFLD is associated with the features of MetS, such as increased blood pressure and also the development of an atherogenic lipoprotein profile. In $\mathrm{NASH}$, key components of the renin-angiotensin-aldosterone system (RAS) are also increased, and RAS activity plays a key role linking NAFLD to vascular disease in CKD. It is well known that adipocytes express all components of RAS contributing up to $30 \%$ of circulating renin, angiotensin-converting enzyme, and the vasoconstrictor angiotensin II, but the liver also expresses RAS constituents, and experimental studies support a role for both systemic and local activation of the angiotensin II in NAFLD ${ }^{140}$.

The specific atherogenic lipoprotein profile that is typically associated with the MetS and insulin resistance increases secretion of triglyceride-rich lipoproteins (TGRLP) into the circulation. With an increase in TGRLPs in the circulation there is also an associated increase in cholesterol ester transfer protein (CETP) activity ${ }^{104,140-142}$. CETP resides on high-density lipoproteins (HDL) and mediates the reciprocal exchange of triglyceride 
and cholesterol esters between TGRLPs and both the HDL and LDL particles. With a CETP-mediated increase in triglyceride content of HDL and LDL lipoproteins, both lipoprotein particles are cleared from the circulation, resulting in increased plasma concentrations of small, dense HDL and small, dense LDL particles. Small dense HDL particles are less efficient in facilitating reverse cholesterol transfer, a process whereby excess cholesterol is cleared from peripheral tissues (including the vasculature) to the liver, and small dense LDL particles are more atherogenic than 'normal' LDL particles $^{140-142}$.

In individuals with MetS, insulin resistance and progressive forms of NAFLD, an upregulation of multiple proinflammatory pathways is almost invariably observed. These proinflammatory mechanisms influence two main intracellular transcription factorsignaling pathways, the nuclear factor-kB (NF-kB) pathway and the C-Jun-N-terminal kinase (JNK) pathway ${ }^{141-143}$. Experimental animal data suggest that JNK-1 activation in the adipose tissue causes insulin resistance in the liver ${ }^{144}$, and activation of the NF-kB pathway in NASH is potentially pivotal in further amplifying the systemic inflammatory response with an NF-kB pathway-mediated increase in transcription of several different proinflammatory genes ${ }^{145}$.

Additionally, adiponectin and leptin are two key adipokines, which may have an impact on disease progression in NAFLD by regulation of hepatic fat accumulation, inflammation and fibrosis ${ }^{145,146}$. Adiponectin exerts anti-inflammatory, anti-fibrotic and anti-atherogenic properties, and therefore low levels of adiponectin are associated with insulin resistance, and NASH may influence progression not only of liver disease and HCC in NAFLD, but also may increase risk of CVD and $C K D^{146}$.

Hepatic lipids that are not esterified are also able to induce endoplasmic reticulum stress, leading to activation of JNK kinase and NF-KB ${ }^{147}$. Hepatic lipid may also induce an endoplasmic reticulum oxidative stress response, as well as induce mitochondrial dysfunction with the potential for generation of free radicals, via increased oxidation of excess fatty acids ${ }^{148}$. Mitochondrial dysfunction is associated with insulin resistance and atherosclerosis in several studies $^{149}$, suggesting a mechanism linking NAFLD and increased risk of CVD and CKD. 
Finally, it is known that the liver is key to the production of multiple coagulation factors and is also an important site of production of plasminogen activator inhibitor-1 (PAl-1) and other fibrinolytic proteins ${ }^{150}$. Several case-control studies have shown that the levels of multiple procoagulant factors (e.g., fibrinogen, factor VII, von Willebrand factor, PAI-1 and other haemostatic-fibrinolytic factors) are highest in patients with NASH, intermediate in those with simple steatosis, and lowest in control subjects without steatosis (as reviewed in ${ }^{151,152}$ ), supporting a "dose-response" relationship between the severity of NAFLD and prothrombotic risk. Studies have also shown that NASH is associated with abnormal intrahepatic expression of most of the above-mentioned proinflammatory and procoagulant biomarkers ${ }^{153,154}$, thus further supporting the notion that the increased circulating levels of these biomarkers result from the up-regulation of their synthesis, taking place in the steatotic /inflamed/fibrotic liver.

Figure 6 shows the more relevant liver-specific pathways potentially linking NAFLD to increased risk of CVD and CKD.

In summary, recent evidence clearly suggests that NAFLD may increase the risk of chronic vascular complications of diabetes via a variety of different pathogenic mechanisms. These biological pathways potentially include dysbiosis and perturbed intestinal function, abdominal obesity, insulin resistance, atherogenic dyslipidaemia, proinflammatory cytokines, increased oxidative stress and alterations of haemostaticfibrinolytic factors. Despite the biological plausibility of dysbiosis and intestinal dysfunction might also represent a novel mediator increasing risk of both NAFLD and vascular disease, presently, it remains uncertain whether treatment for dysbiosis favourably modifies levels of potentially damaging molecules and pathways leading to NAFLD and to chronic vascular and renal damage. Although further research is urgently needed in this area, correction of dysbiosis might represent a novel therapeutic target to ameliorate both risk of NAFLD and also CVD (and CKD) ${ }^{108,126,155 .}$

\section{MANAGEMENT AND TREATMENT OPTIONS FOR NAFLD IN DIABETES}

\subsection{Lifestyle modification}

The therapeutic approach to patients with coexistent NAFLD and diabetes should be multifactorial. Currently, the mainstay of NAFLD management in these patients is to reduce body weight, improve glycaemic control and reduce the modifiable 
cardiometabolic risk factors (possibly using drugs with potentially beneficial effects on the liver $)^{1,2,45,47,156}$.

Lifestyle changes must be suggested to all patients with coexistent NAFLD and diabetes even though they are difficult to achieve and maintain. Moreover, a strict control of all coexisting cardiometabolic risk factors should be pursued and the higher the risk of progressive liver disease, the more aggressive the treatment should be. However, to date, whether patients with coexistent NAFLD and diabetes should be treated to a specific haemoglobin A1c, LDL-cholesterol, and blood pressure target remains uncertain.

\subsubsection{Body weight reduction}

The importance of losing body weight in patients with T2DM cannot be overemphasized and, in those with $\mathrm{NASH}$, a weight reduction of nearly $5-7 \%$ is able to decrease hepatic steatosis; however, a nearly $8-10 \%$ weight reduction is requested to reverse NASH and a weight loss of $\geq 10 \%$ can also improve or reverse hepatic fibrosis ${ }^{1,2,156,157}$. Given the difficulties in achieving and maintaining this endpoint through lifestyle modifications, bariatric surgery, which is able to improve all histological lesions of $\mathrm{NASH}$, including hepatic fibrosis, may be suggested to properly selected severely obese patients $^{1,2,45,158,159}$. However, whilst bariatric surgery is undoubtedly effective, there are obvious limitations, including possible complications, patient compliance, service availability and cost.

\subsubsection{Diet and smoking}

Qualitative and quantitative dietary changes are advisable for all patients with NAFLD ${ }^{1,2}$. About 1200-1600 kcal/day are recommended. A low-fat (<30\%) diet with less than 10\% of saturated fatty acid intake, and relatively low in carbohydrate intakes $(<50 \%$ of total Kcals) is suggested. Preference should be given to complex carbohydrates, avoiding simple ones, which have a higher glycemic index; as well as the lipogenic sugar fructose ${ }^{160}$.

Mediterranean diet appears to be the most useful non-pharmacological option aimed at losing body weight while gaining some beneficial effects on cardiometabolic outcomes $^{1,2,161}$. A high dietary intake in fish and vegetables (but not in fruits) has been 
also associated with a protection from developing HCC. Conversely, cigarette smoking and even modest amount of alcohol consumption may increase HCC risk $^{162-164}$.

\subsubsection{Physical exercise}

Both aerobic training and resistance training may reduce hepatic steatosis, independent of weight loss $1,2,45,156,165$. Physical exercise should be individualized based on the patient's attitude and convenience and, ideally, maintained indefinitely ${ }^{165-167}$. Since patients with NAFLD are often at a high risk of developing CVD ${ }^{168}$, a careful cardiac evaluation may prudently be implemented in these patients before submitting them to any vigorous physical efforts.

\subsection{Pharmacological treatment}

A detailed review of drug treatment options for NAFLD/NASH in patients with diabetes is beyond the scope of this article. Comprehensive review articles of this topic have been published elsewhere ${ }^{1,2,45,56,156,169}$.

Currently, there are no licensed pharmacological agents specifically for the treatment of NAFLD. The major issue in this field is the scarcity of high-quality, randomized, blinded, adequately powered, controlled trials of sufficient duration and with clinically relevant endpoints. In line with this consideration, a recent Cochrane review ${ }^{170}$ concluded that "we are very uncertain about the effectiveness of pharmacological treatments for people with NAFLD, including those with NASH". Some concerns also remain about the longterm safety of the available drugs, necessitating thoughtful balancing of the potential risks and benefits.

Therefore, to date, drug treatment is best reserved to those patients with NASH, who are at the highest risk of progressive liver disease and/or those patients who have more severely decompensated diabetes ${ }^{1,2,45,56,156,169}$.

A central dogma is that any diabetes treatment may benefit patients with NASH if they have uncontrolled hyperglycaemia ${ }^{45,56}$. Further to glucose-lowering agents, several classes of drugs can be taken into consideration, such as lipid-lowering agents; antioxidants; iron depletion; innovative drugs and, in some cases, bariatric (metabolic) surgery ${ }^{1,2,45,56,156,169}$. A variety of drugs will also probably emerge over the next 5 years, 
permitting a more stage-based approach of NAFLD and greater personalization of drug selection.

Table 1 summarizes the effects on liver histology of drug treatments for NASH observed in the principal randomized clinical trials that included adults with T2DM or prediabetes with biopsy-proven NASH ${ }^{131,171-181}$.

In choosing among the various available drug classes for the treatment of patients with coexistent NAFLD and T2DM, we believe that priority should be given to those drug principles whose action is not limited to an individual therapeutic target but is also extended to improve the risk of developing CVD events and severe liver-related complications, such as cirrhosis and $\mathrm{HCC}^{168}$. Statins are an example of such a pleiotropic class of drugs ${ }^{182}$. For instance, a cross-sectional survey conducted in nearly 350 patients with T2DM and histologically proven NAFLD has shown that the use of statins is inversely associated, while the use of insulin or sulphonylureas appear to be positively associated with the presence and severity of $\mathrm{NASH}$ and fibrosis on liver histology ${ }^{183}$. Similarly, the use of pioglitazone, which has to date the most available evidence of efficacy in T2DM patients with $\mathrm{NASH}$, may potentially improve the natural history of the liver disease by reducing its progression to cirrhosis in (some) patients with biopsy-proven $\mathrm{NASH}^{1,2,45,56,156,169}$. Conversely, metformin is not currently recommended as a specific treatment for liver disease in T2DM patients with NAFLD or $\mathrm{NASH}$. Furthermore, there are no robust data with clinically relevant endpoints as a primary outcome to formally comment on the effectiveness of the use of the newer glucose-lowering agents (e.g., dipeptidyl peptidase-4 inhibitors, glucagon-like peptide-1 receptor agonists or sodium glucose co-transporter 2 inhibitors) as a treatment for NAFLD/NASH with coexistent diabetes. On these grounds, an Expert Panel recently suggested that, pending forthcoming randomized clinical trials, physicians should consider using pioglitazone, or, statin use in those patients with NAFLD/NASH at high CVD or HCC risk (unless contraindicated), alone or preferably in combination with each other or with ezetimibe, for the primary or secondary prevention of CVD, and the avoidance of cirrhosis, liver transplantation or HCC, bearing in mind that CVD is the main cause of death in patients with NAFLD ${ }^{184}$. In 2016, the UK National Institute for Care and Clinical Excellence (NICE) recommended that in secondary or tertiary care settings only, clinicians should consider treatment with pioglitazone or vitamin $E$ for 
adults with advanced liver fibrosis (whether they have diabetes or not). Before prescribing pioglitazone or vitamin $\mathrm{E}$, it was recommended that clinicians take into account any comorbidities and the risk of adverse events associated with these conditions ${ }^{185}$. However, that said, it is important to note that for all treatments that have been advocated for NAFLD, not all patients respond to treatment. Given that all available treatments have potentially harmful side effects, until there are accepted strategies for monitoring responses to therapy, it is difficult to advocate that a treatment be started if there is no regular monitoring of treatment efficacy.

\section{CONCLUSIONS}

Existing guidelines do not advocate screening for liver-related complications in patients with T2DM or T1DM, making the liver a potentially neglected target organ for undetected chronic disease progression to cirrhosis. However, given the increasingly growing prevalence and incidence of NAFLD in patients with diabetes and its related hepatic and extra-hepatic complications, NAFLD should always be ruled out in adult patients with T2DM or T1DM.

This review article supports the existence of a strong association between the presence and severity of NAFLD and the risk of chronic vascular complications of diabetes, mainly CVD, cardiomyopathy (left ventricular dysfunction and hypertrophy) and CKD. Despite the growing evidence that links NAFLD with CVD, CKD and other microvascular complications of diabetes, it remains to be definitively established whether a causal association also exists.

In the meantime, however, these findings call for a more active and systematic search for NAFLD in adult patients with T2DM or T1DM with a view to potential earlier treatment. We strongly believe that the possibility of NAFLD should be entertained as a part of the routine evaluation of adult patients with T2DM or T1DM, in the same way we search for CVD, CKD and other chronic complications of diabetes. 


\section{KEY POINTS}

- Convincing epidemiological evidence substantiates a strong association between the presence and severity of NAFLD and risk of chronic macrovascular (mainly cardiovascular disease) and microvascular (mainly chronic kidney disease) complications of diabetes.

- Evidence suggests that NAFLD exacerbates insulin resistance, predisposes to atherogenic dyslipidaemia and causes the release of a variety of proinflammatory, procoagulant and proatherogenic mediators that play a role in the development of chronic vascular complications of diabetes.

- Despite the evidence linking NAFLD to these chronic vascular complications, it has not been definitively established whether a causal association also exists.

- These findings call for a more active and systematic search for NAFLD in adult patients with diabetes (with the combined use of serum liver enzymes, liver ultrasonography, FibroScan and non-invasive tests of advanced hepatic fibrosis) with a view to implementing an earlier and more aggressive treatment whenever indicated.

- Whether such a more liberal screening policy and more aggressive treatment will costeffectively prevent the development of chronic vascular complications of diabetes will be the target of future larger studies.

\section{Acknowledgements}

G.T. is supported in part by grants from the University School of Medicine of Verona, Verona, Italy. C.D.B. is supported in part by the Southampton National Institute for Health Research Biomedical Research Centre.

\section{Competing interests statement}

G.T. \& C.D.B. declare no competing financial interests. A.L. is a researcher of a phase III, double-blind, randomized, long-term, placebo controlled, multicenter study evaluating the safety and efficacy of obeticholic acid in subjects with NASH (EudraCT 2015-002560-16).

\section{Author Contributions}


All authors researched the data for the article, provided substantial contributions to discussions of its content, wrote the article and undertook review and/or editing of the manuscript before submission.

\section{Author biographies}

Giovanni Targher, M.D. is an Associate Professor and Senior Consultant at the Section of Endocrinology, Diabetes and Metabolism, Department of Medicine, University of Verona, and at Azienda Ospedaliera Universitaria Integrata, Verona, Italy. His main research interests are NAFLD and its relationships with cardiovascular disease, chronic kidney disease and other extra-hepatic complications.

Amedeo Lonardo, M.D. is Vice-director of the Complex Operating Unit of Internal Medicine at the Ospedale Civile di Baggiovara, Azienda Ospedaliero-Universitaria of Modena, Italy. His main research interests are the hepatic and extra-hepatic outcomes of fatty liver disorders due to varying etiologies. In 2017, he has been acknowledged suitable as Full Professor of Gastroenterology and Associate Professor in Internal Medicine and Gastroenterology at the National Italian Examination.

Christopher D. Byrne M.B. BCh. is Chair of Endocrinology \& Metabolism at the University of Southampton, UK and Principal Investigator within the Southampton NIHR Biomedical Research Centre. He specializes in the management of patients with diabetes and liver disease, and was Expert Diabetologist Advisor and Panel member to the UK National Institute for Care Excellence (NICE) NAFLD Guideline Development Group. He has published extensively on NAFLD and its pathogenesis, extra-hepatic complications, and treatments.

\section{REFERENCES}

1. European Association for the Study of the Liver (EASL); European Association for the Study of Diabetes (EASD); European Association for the Study of Obesity (EASO). EASL-EASD-EASO clinical practice guidelines for the management of non-alcoholic fatty liver disease. J. Hepatol. 64, 1388-1402 (2016).

2. Italian Association for the Study of the Liver (AISF). AISF position paper on nonalcoholic fatty liver disease (NAFLD): updates and future directions. Dig. Liver Dis. 49, 471-483 (2017). 
3. Anstee, Q.M., Targher, G. \& Day, C.P. Progression of NAFLD to diabetes mellitus, cardiovascular disease or cirrhosis. Nat. Rev. Gastroenterol. Hepatol. 10, 330-344 (2013).

4. Byrne, C.D. \& Targher, G. NAFLD: a multisystem disease. J. Hepatol. 62, S47S64 (2015).

5. Armstrong, M.J., Adams, L.A., Canbay, A. \& Syn, W.K. Extrahepatic complications of nonalcoholic fatty liver disease. Hepatology 59, 1174-1197 (2014).

6. Adams, L.A., Anstee, Q.M., Tilg, H. \& Targher, G. Non-alcoholic fatty liver disease and its relationship with cardiovascular disease and other extrahepatic diseases. Gut 66, 1138-1153 (2017).

7. Loria, P., Lonardo, A. \& Carulli, N. Should nonalcoholic fatty liver disease be renamed? Dig. Dis. 23, $72-78$ (2005).

8. Brunt, E.M. What's in a NAme? Hepatology 50, 663-667 (2009).

9. Nascimbeni, F. et al. From NAFLD in clinical practice to answers from guidelines. J. Hepatol. 59, 859-871 (2013).

10. Bugianesi, E., Rosso, C. \& Cortez-Pinto, H. How to diagnose NAFLD in 2016. J. Hepatol. 65, 643-644 (2016).

11. Lonardo, A. et al. Epidemiological modifiers of non-alcoholic fatty liver disease: Focus on high-risk groups. Dig. Liver Dis. 47, 997-1006 (2015).

12. Fedchuk, L. et al. Performance and limitations of steatosis biomarkers in patients with nonalcoholic fatty liver disease. Aliment. Pharmacol. Ther. 40, 1209-1222 (2014).

13. Hernaez, R. et al. Diagnostic accuracy and reliability of ultrasonography for the detection of fatty liver: a meta-analysis. Hepatology 54,1082-1090 (2011).

14. Ballestri, S., Romagnoli, D., Nascimbeni, F., Francica, G. \& Lonardo, A. Role of ultrasound in the diagnosis and treatment of nonalcoholic fatty liver disease and its complications. Expert Rev. Gastroenterol. Hepatol. 9, 603-627 (2015).

15. Ballestri, S. et al. Ultrasonographic fatty liver indicator detects mild steatosis and correlates with metabolic/histological parameters in various liver diseases. Metabolism 72, 57-65 (2017).

16. Ballestri, S. et al. Ultrasonographic fatty liver indicator, a novel score which rules out NASH and is correlated with metabolic parameters in NAFLD. Liver Int. 32, 1242-1252 (2012).

17. de Lédinghen, V. et al. Controlled attenuation parameter for the diagnosis of steatosis in non-alcoholic fatty liver disease. J. Gastroenterol. Hepatol. 31, 848855 (2016).

18. Spithoff, S. \& Kahan. M. Primary care management of alcohol use disorder and at-risk drinking: Part 1: screening and assessment. Can. Fam. Physician. 61, 509-514 (2015).

19. Yang, K.C. et al. Association of non-alcoholic fatty liver disease with metabolic syndrome independently of central obesity and insulin resistance. Sci. Rep. 6, 27034 (2016).

20. Nascimbeni, F. et al. Clinical relevance of liver histopathology and different histologic classifications of NASH in adults. Expert Review Gastroenterol. Hepatol. in press.

21. Petta, S. et al. Serial combination of non-invasive tools improves the diagnostic accuracy of severe liver fibrosis in patients with NAFLD. Aliment. Pharmacol. Ther. 2017 Jul 27. doi: 10.1111/apt.14219 [Epub ahead of print]. 
22. Chalasani, N. et al. The diagnosis and management of nonalcoholic fatty liver disease: practice guidance from the american association for the study of liver diseases. Hepatology 2017 Jul 17. doi: 10.1002/hep.29367 [Epub ahead of print].

23. Wong, V.W. et al. The Asia-Pacific working party on nonalcoholic fatty liver disease guidelines 2017 part 1: definition, risk factors and assessment. J .Gastroenterol. Hepatol. 2017 Jul 3. doi: 10.1111/jgh.13857 [Epub ahead of print].

24. Corey, K.E., Klebanoff, M.J., Tramontano, A.C., Chung, R.T. \& Hur, C. Screening for nonalcoholic steatohepatitis in individuals with type 2 diabetes: a costeffectiveness analysis. Dig. Dis. Sci. 61, 2108-2117 (2016).

25. Phisalprapa, P. et al. Cost-effectiveness analysis of ultrasonography screening for nonalcoholic fatty liver disease in metabolic syndrome patients. Medicine (Baltimore) 96, e6585 (2017).

26. Singh, A., Le, P., Peerzada, M.M., Lopez, R. \& Alkhouri, N. The utility of noninvasive scores in assessing the prevalence of nonalcoholic fatty liver disease and advanced fibrosis in type 2 diabetic patients. J. Clin. Gastroenterol. 2017 Aug 4. doi: 10.1097/MCG.0000000000000905 [Epub ahead of print].

27. Younossi, Z.M. et al. Global epidemiology of nonalcoholic fatty liver disease Meta-analytic assessment of prevalence, incidence, and outcomes. Hepatology 64, 73-84 (2016).

28. Targher, G. et al. Prevalence of nonalcoholic fatty liver disease and its association with cardiovascular disease among type 2 diabetic patients. Diabetes Care 30, 1212-1218 (2007).

29.Leite, N.C., Salles, G.F., Araujo, A.L., Villela-Nogueira, C.A. \& Cardoso, C.R. Prevalence and associated factors of non-alcoholic fatty liver disease in patients with type-2 diabetes mellitus. Liver Int. 29, 113-119 (2009).

30. Leite, N.C. et al. Histopathological stages of nonalcoholic fatty liver disease in type 2 diabetes: prevalences and correlated factors. Liver Int. 31, 700-706 (2011).

31. Williamson, R.M. et al. Prevalence of and risk factors for hepatic steatosis and nonalcoholic fatty liver disease in people with type 2 diabetes: the Edinburgh Type 2 Diabetes Study. Diabetes Care 34, 1139-1144 (2011).

32. Kim, S.K. et al. Nonalcoholic fatty liver disease is associated with increased carotid intima-media thickness only in type 2 diabetic subjects with insulin resistance. J. Clin. Endocrinol. Metab. 99, 1879-1884 (2014).

33. Portillo-Sanchez, P. et al. High prevalence of nonalcoholic fatty liver disease in patients with type 2 diabetes mellitus and normal plasma aminotransferase levels. J. Clin. Endocrinol. Metab. 100, 2231-2238 (2015).

34. Kwok, R. et al. Screening diabetic patients for non-alcoholic fatty liver disease with controlled attenuation parameter and liver stiffness measurements: a prospective cohort study. Gut 65, 1359-1368 (2016).

35. Masarone, M. et al. Liver biopsy in type 2 diabetes mellitus: Steatohepatitis represents the sole feature of liver damage. PLoS One 12, e0178473 (2017).

36. Browning, J.D. et al. Prevalence of hepatic steatosis in an urban population in the United States: impact of ethnicity. Hepatology 40, 1387-1395 (2004).

37. Volzke, H. et al. Hepatic steatosis is associated with an increased risk of carotid atherosclerosis. World J. Gastroenterol. 11, 1848-1853 (2005).

38. Jimba, S. et al. Prevalence of non-alcoholic fatty liver disease and its association with impaired glucose metabolism in Japanese adults. Diabet. Med. 22, 11411145 (2005). 
39. Speliotes, E.K. et al. Fatty liver is associated with dyslipidemia and dysglycemia independent of visceral fat: the Framingham Heart Study. Hepatology 51, 19791987 (2010).

40.Williams, C.D. et al. Prevalence of nonalcoholic fatty liver disease and nonalcoholic steatohepatitis among a largely middle-aged population utilizing ultrasound and liver biopsy: a prospective study. Gastroenterology 140, 124-113 (2011).

41. Lazo, M. et al. Prevalence of nonalcoholic fatty liver disease in the United States: the Third National Health and Nutrition Examination Survey, 1988-1994. Am. J. Epidemiol. 178, 38-45 (2013).

42.Zeb, I. et al. Relation of nonalcoholic fatty liver disease to the metabolic syndrome: the Multi-Ethnic Study of Atherosclerosis. J. Cardiovasc. Comput. Tomogr. 7, 311-318 (2013).

43. Wild, S.H. et al.; Scottish and Southampton Diabetes and Liver Disease Group; Scottish Diabetes Research Network Epidemiology Group. Type 2 diabetes and risk of hospital admission or death for chronic liver diseases. J. Hepatol. 64, 1358-1364 (2016).

44. Wilman, H.R. et al. Characterisation of liver fat in the UK Biobank cohort. PLoS One 12, e0172921 (2017).

45. Bril, F. \& Cusi, K. Management of nonalcoholic fatty liver disease in patients with type 2 diabetes: a call to action. Diabetes Care 40, 419-430 (2017).

46. Loria, P., Lonardo, A. \& Anania, F. Liver and diabetes. A vicious circle. Hepatol. Res. 43, 51-64 (2013).

47. Targher, G. \& Byrne, C.D. Clinical review: nonalcoholic fatty liver disease: a novel cardiometabolic risk factor for type 2 diabetes and its complications. $J$. Clin. Endocrinol. Metab. 98, 483-495 (2013).

48. Harris, R., Harman, D.J., Card, T.R., Aithal, G.P. \& Guha, I.N. Prevalence of clinically significant liver disease within the general population, as defined by non-invasive markers of liver fibrosis: a systematic review. Lancet Gastroenterol. Hepatol. 2, 288-297 (2017).

49. Valenti, L., Bugianesi, E., Pajvani, U. \& Targher, G. Nonalcoholic fatty liver disease: cause or consequence of type 2 diabetes? Liver Int. 36, 1563-1579 (2016).

50.Porepa, L., Ray, J.G., Sanchez-Romeu, P. \& Booth, G.L. Newly diagnosed diabetes mellitus as a risk factor for serious liver disease. C.M.A.J. 182, E526E531 (2010).

51.Zoppini, G. et al. Mortality from chronic liver diseases in diabetes. Am. J. Gastroenterol. 109, 1020-1025 (2014).

52. Michelotti, G.A., Machado, M.V. \& Diehl, A.M. NAFLD, NASH and liver cancer. Nat. Rev. Gastroenterol. Hepatol. 10, 656-665 (2013).

53. Dyson, J. et al. Hepatocellular cancer: the impact of obesity, type 2 diabetes and a multidisciplinary team. J. Hepatol. 60, 110-117 (2014).

54. Singal, A.G. \& El-Serag, H.B. Hepatocellular carcinoma from epidemiology to prevention: translating knowledge into practice. Clin. Gastroenterol. Hepatol. 13, 2140-2151 (2015).

55. Piscaglia, F. et al.; HCC-NAFLD Italian Study Group. Clinical patterns of hepatocellular carcinoma in nonalcoholic fatty liver disease: a multicenter prospective study. Hepatology 63, 827-838 (2016).

56. Targher, G. \& Byrne, C.D. Non-alcoholic fatty liver disease: an emerging driving force in chronic kidney disease. Nat. Rev. Nephrol. 13, 297-310 (2017). 
57. Targher, G., Byrne, C.D., Lonardo, A., Zoppini, G. \& Barbui, C. Non-alcoholic fatty liver disease and risk of incident cardiovascular disease: a meta-analysis. $J$. Hepatol. 65, 589-600 (2016).

58. Lonardo, A., Sookoian, S., Pirola, C.J. \& Targher, G. Non-alcoholic fatty liver disease and risk of cardiovascular disease. Metabolism 65, 1136-1150 (2016).

59. Leeds, J.S. et al. Abnormal liver function tests in patients with type 1 diabetes mellitus: prevalence, clinical correlations and underlying pathologies. Diabet. Med. 26, 1235-1241 (2009).

60. Targher, G. et al. Prevalence of non-alcoholic fatty liver disease and its association with cardiovascular disease in patients with type 1 diabetes. $J$. Hepatol. 53, 713-718 (2010).

61.Targher, G., Pichiri, I., Zoppini, G., Trombetta, M. \& Bonora, E. Increased prevalence of cardiovascular disease in type 1 diabetic patients with nonalcoholic fatty liver disease. J. Endocrinol. Invest. 35, 535-540 (2012).

62. Elkabbany, Z.A. et al. Transient elastography as a noninvasive assessment tool for hepatopathies of different etiology in pediatric type 1 diabetes mellitus. $J$. Diabetes Complications 31, 186-194 (2017).

63. Regnell, S.E. et al. Magnetic resonance imaging reveals altered distribution of hepatic fat in children with type 1 diabetes compared to controls. Metabolism 64, 872-878 (2015).

64. Llauradó, G. et al. Liver fat content and hepatic insulin sensitivity in overweight patients with type 1 diabetes. J. Clin. Endocrinol. Metab. 100, 607-616 (2015).

65. Petit, J.M. et al. Type 1 diabetes is not associated with an increased prevalence of hepatic steatosis. Diabet. Med. 32, 1648-1651 (2015).

66. Cusi, K. et al. Non-alcoholic fatty liver disease (NAFLD) prevalence and its metabolic associations in patients with type 1 diabetes and type 2 diabetes. Diabetes Obes. Metab. 2017 Apr 17. doi: 10.1111/dom.12973 [Epub ahead of print].

67. Sviklāne, L. et al. Fatty liver index and hepatic steatosis index predict nonalcoholic fatty liver disease in type 1 diabetes. J. Gastroenterol. Hepatol. 2017 May 2. doi: 10.1111/jgh.13814 [Epub ahead of print].

68. Harman, D.J. et al. Prevalence and natural history of histologically proven chronic liver disease in a longitudinal cohort of patients with type 1 diabetes. Hepatology 60, 158-168 (2014).

69. Targher, G., Day, C.P. \& Bonora, E. Risk of cardiovascular disease in patients with nonalcoholic fatty liver disease. N. Engl. J. Med. 363, 1341-1350 (2010).

70. Mantovani, A., Ballestri, S., Lonardo, A. \& Targher, G. Cardiovascular disease and myocardial abnormalities in nonalcoholic fatty liver disease. Dig. Dis. Sci. 61, 1246-1267 (2016).

71. Guo, K. et al. Non-alcoholic fatty liver disease is associated with late but not early atherosclerotic lesions in Chinese inpatients with type 2 diabetes. $J$. Diabetes Complications 31, 80-85 (2017).

72. Wong, V.W. et al. Coronary artery disease and cardiovascular outcomes in patients with non-alcoholic fatty liver disease. Gut 60, 1721-1727 (2011).

73. Idilman, I.S. et al. Nonalcoholic fatty liver disease is associated with significant coronary artery disease in type 2 diabetic patients: a computed tomography angiography study. J. Diabetes 7, 279-286 (2015).

74. Puchner, S.B. et al. High-risk coronary plaque at coronary CT angiography is associated with nonalcoholic fatty liver disease, independent of coronary plaque and stenosis burden: results from the ROMICAT II trial. Radiology 274, 693-701 (2015). 
75. Bonapace, S. et al. Nonalcoholic fatty liver disease is associated with left ventricular diastolic dysfunction in patients with type 2 diabetes. Diabetes Care 35, 389-395 (2012).

76. Mantovani, A. et al. Nonalcoholic fatty liver disease is independently associated with early left ventricular diastolic dysfunction in patients with type 2 diabetes. PLoS One 10, e0135329 (2015).

77. Mantovani, A., Zoppini, G., Targher, G., Golia, G. \& Bonora, E. Non-alcoholic fatty liver disease is independently associated with left ventricular hypertrophy in hypertensive type 2 diabetic individuals. J. Endocrinol. Invest. 35, 215-218 (2012).

78. Mantovani, A. et al. Heart valve calcification in patients with type 2 diabetes and nonalcoholic fatty liver disease. Metabolism 64, 879-887 (2015).

79. Targher, G. et al. Non-alcoholic fatty liver disease is associated with an increased prevalence of atrial fibrillation in hospitalized patients with type 2 diabetes. Clin. Sci. (Lond) 125, 301-309 (2013).

80. Targher, G. et al. Non-alcoholic fatty liver disease is associated with an increased incidence of atrial fibrillation in patients with type 2 diabetes. PLoS One 8, e57183 (2013).

81. VanWagner, L.B. et al. Association of nonalcoholic fatty liver disease with subclinical myocardial remodeling and dysfunction: a population-based study. Hepatology 62, 773-783 (2015).

82. VanWagner L.B. \& Rinella M.E. Extrahepatic manifestations of nonalcoholic fatty liver disease. Curr. Hepatol. Rep. 15, 75-85 (2016).

83. Targher, G. et al. Association of nonalcoholic fatty liver disease with QTc interval in patients with type 2 diabetes. Nutr. Metab. Cardiovasc. Dis. 24, 663-669 (2014).

84. Mantovani, A. et al. Nonalcoholic fatty liver disease is associated with ventricular arrhythmias in patients with type 2 diabetes referred for clinically indicated 24hour holter monitoring. Diabetes Care 39, 1416-1423 (2016).

85. Valbusa, F. et al. Nonalcoholic fatty liver disease and increased risk of 1-year allcause and cardiac hospital readmissions in elderly patients admitted for acute heart failure. PLoS One 12, e0173398 (2017).

86. Targher, G. et al. Nonalcoholic fatty liver disease and risk of future cardiovascular events among type 2 diabetic patients. Diabetes 54, 3541-3546 (2005).

87. Targher, G. et al. Nonalcoholic fatty liver disease is independently associated with an increased incidence of cardiovascular events in type 2 diabetic patients. Diabetes Care 30, 2119-2221 (2007).

88. Mantovani, A. et al. Nonalcoholic fatty liver disease is independently associated with an increased incidence of cardiovascular disease in adult patients with type 1 diabetes. Int. J. Cardiol. 225, 387-391 (2016).

89. Targher G., Chonchol M.B. \& Byrne C.D. CKD and nonalcoholic fatty liver disease. Am. J. Kidney Dis. 64, 638-652 (2014).

90. Targher, G. et al. Non-alcoholic fatty liver disease is independently associated with an increased prevalence of chronic kidney disease and proliferative/lasertreated retinopathy in type 2 diabetic patients. Diabetologia 51, 444-450 (2008).

91.Lin, T.Y., Chen, Y.J., Chen, W.L. \& Peng, T.C. The relationship between nonalcoholic fatty liver disease and retinopathy in NHANES III. PLoS One 11, e0165970 (2016). 
92. Li, Y. et al. Association between non-alcoholic fatty liver disease and chronic kidney disease in population with prediabetes or diabetes. Int. Urol. Nephrol. 46, 1785-1791 (2014).

93. Targher, G. et al. Relationship between kidney function and liver histology in subjects with nonalcoholic steatohepatitis. Clin. J. Am. Soc. Nephrol. 5, 21662171 (2010).

94. Targher, G. et al. Non-alcoholic fatty liver disease is independently associated with an increased prevalence of chronic kidney disease and retinopathy in type 1 diabetic patients. Diabetologia 53, 1341-1348 (2010).

95. Targher, G., Pichiri, I., Zoppini, G., Trombetta, M. \& Bonora, E. Increased prevalence of chronic kidney disease in patients with type 1 diabetes and nonalcoholic fatty liver. Diabet. Med. 29, 220-226 (2012).

96. Targher, G. et al. Increased risk of CKD among type 2 diabetics with nonalcoholic fatty liver disease. J. Am. Soc. Nephrol. 19, 1564-1570 (2008).

97. Targher, G. et al. Nonalcoholic fatty liver disease is independently associated with an increased incidence of chronic kidney disease in patients with type 1 diabetes. Diabetes Care 37, 1729-1736 (2014).

98. Musso, G. et al. Association of non-alcoholic fatty liver disease with chronic kidney disease: a systematic review and meta-analysis. PLoS Med. 11, e1001680 (2014).

99. Mantovani, A. et al. Nonalcoholic fatty liver disease is associated with an increased prevalence of distal symmetric polyneuropathy in adult patients with type 1 diabetes. J. Diabetes Complications 31, 1021-1026 (2017).

100. Williams, K.H. et al. An association of large-fibre peripheral nerve dysfunction with non-invasive measures of liver fibrosis secondary to nonalcoholic fatty liver disease in diabetes. J. Diabetes Complications 29, 1240-1247 (2015).

101. Kim, B.Y., Jung, C.H., Mok, J.O., Kang, S.K. \& Kim, C.H. Prevalences of diabetic retinopathy and nephropathy are lower in Korean type 2 diabetic patients with non-alcoholic fatty liver disease. J. Diabetes Invest. 5, 170-175 (2014).

102. Lv, W.S. et al. Nonalcoholic fatty liver disease and microvascular complications in type 2 diabetes. World J. Gastroenterol. 19, 3134-3142 (2013).

103. Stern, M.P. Diabetes and cardiovascular disease. The "common soil" hypothesis. Diabetes 44, 369-374 (1995).

104. DeFronzo, R.A. Banting Lecture. From the triumvirate to the ominous octet: a new paradigm for the treatment of type 2 diabetes mellitus. Diabetes 58, 773-795 (2009).

105. Alberti, K.G. et al. Harmonizing the metabolic syndrome: a joint interim statement of the International Diabetes Federation Task Force on Epidemiology and Prevention; National Heart, Lung, and Blood Institute; American Heart Association; World Heart Federation; International Atherosclerosis Society; and international association for the Study of Obesity. Circulation 120, 1640-1645 (2009).

106. Zhu, W. et al. Gut microbial metabolite tmao enhances platelet hyperreactivity and thrombosis risk. Cell 165, 111-124 (2016).

107. Wilson, A., McLean, C. \& Kim, R.B. Trimethylamine-N-oxide: a link between the gut microbiome, bile acid metabolism, and atherosclerosis. Curr. Opin. Lipidol. 27, 148-154 (2016).

108. Koopen, A.M., Groen, A.K. \& Nieuwdorp, M. Human microbiome as therapeutic intervention target to reduce cardiovascular disease risk. Curr. Opin. Lipidol. 27, 615-622 (2016). 
109. Brown, J.M. \& Hazen, S.L. The gut microbial endocrine organ: bacterially derived signals driving cardiometabolic diseases. Annu. Rev. Med. 66, 343-359 (2015).

110. Tang, W.H. et al. Intestinal microbial metabolism of phosphatidylcholine and cardiovascular risk. N. Engl. J. Med. 368, 1575-1584 (2013).

111. Mehal, W.Z. The Gordian knot of dysbiosis, obesity and NAFLD. Nat. Rev. Gastroenterol. Hepatol. 10, 637-644 (2013).

112. Karlsson, F.H. et al. Gut metagenome in European women with normal, impaired and diabetic glucose control. Nature 498, 99-103 (2013).

113. Qin, J. et al. A metagenome-wide association study of gut microbiota in type 2 diabetes. Nature 490, 55-60 (2012).

114. Larsen, N. et al. Gut microbiota in human adults with type 2 diabetes differs from non-diabetic adults. PLoS One 5, e9085 (2010).

115. Utzschneider, K.M., Kratz, M., Damman, C.J. \& Hullarg, M. Mechanisms Linking the Gut Microbiome and Glucose Metabolism. J. Clin. Endocrinol. Metab. 101, 1445-1454 (2016).

116. Wieland, A., Frank, D.N., Harnke, B. \& Bambha, K. Systematic review: microbial dysbiosis and nonalcoholic fatty liver disease. Aliment. Pharmacol. Ther. 42, 1051-1063 (2015).

117. Boursier, J. et al. The severity of nonalcoholic fatty liver disease is associated with gut dysbiosis and shift in the metabolic function of the gut microbiota. Hepatology 63, 764-775 (2016).

118. Tilg, H., Cani, P.D. \& Mayer, E.A. Gut microbiome and liver diseases. Gut 65, 2035-2044 (2016).

119. Briskey, D., Tucker, P.S., Johnson, D.W. \& Coombes, J.S. Microbiota and the nitrogen cycle: Implications in the development and progression of CVD and CKD. Nitric Oxide 57, 64-70 (2016).

120. Ilan, Y. Leaky gut and the liver: a role for bacterial translocation in nonalcoholic steatohepatitis. World J. Gastroenterol. 18, 2609-2618 (2012).

121. Miele, L. et al. Increased intestinal permeability and tight junction alterations in nonalcoholic fatty liver disease. Hepatology 49, 1877-1887 (2009).

122. Ley, R.E. Obesity and the human microbiome. Curr. Opin. Gastroenterol. 26, 5-11 (2010).

123. Ley, R.E., Turnbaugh, P.J., Klein, S. \& Gordon, J.I. Microbial ecology: human gut microbes associated with obesity. Nature 444, 1022-1023 (2006).

124. Endo, H., Niioka, M., Kobayashi, N., Tanaka, M. \& Watanabe, T. Butyrateproducing probiotics reduce nonalcoholic fatty liver disease progression in rats: new insight into the probiotics for the gut-liver axis. PLoS One 8, e63388 (2013).

125. den Besten, G. et al. The role of short-chain fatty acids in the interplay between diet, gut microbiota, and host energy metabolism. J. Lipid Res. 54, 2325-2340 (2013).

126. Beserra, B.T. et al. A systematic review and meta-analysis of the prebiotics and synbiotics effects on glycaemia, insulin concentrations and lipid parameters in adult patients with overweight or obesity. Clin. Nutr. 34, 845-858 (2015).

127. Fernandez-Prado, R. et al. Nutrients turned into toxins: microbiota modulation of nutrient properties in chronic kidney disease. Nutrients 9 (2017).

128. Randrianarisoa, E. et al. Relationship of serum trimethylamine n-oxide (tmao) levels with early atherosclerosis in humans. Sci. Rep. 6, 26745 (2016).

129. Khurana, S., Raufman, J.P. \& Pallone, T.L. Bile acids regulate cardiovascular function. Clin. Transl. Sci. 4, 210-218 (2011). 
130. Xu, J.Y., Li, Z.P., Zhang, L. \& Ji, G. Recent insights into farnesoid X receptor in non-alcoholic fatty liver disease. World J. Gastroenterol. 20, 1349313500 (2014).

131. Neuschwander-Tetri, B.A. et al. Farnesoid $X$ nuclear receptor ligand obeticholic acid for non-cirrhotic, non-alcoholic steatohepatitis (FLINT): a multicentre, randomised, placebo-controlled trial. Lancet 385, 956-965 (2015).

132. lannelli, $\mathrm{F}$. et al. Massive gene amplification drives paediatric hepatocellular carcinoma caused by bile salt export pump deficiency. Nat. Commun. 5, 3850 (2014).

133. Arab, J.P., Karpen, S.J., Dawson, P.A., Arrese, M. \& Trauner, M. Bile acids and nonalcoholic fatty liver disease: Molecular insights and therapeutic perspectives. Hepatology 65, 350-362 (2017).

134. Li, C., Li, J., Weng, X., Lan, X. \& Chi, X. Farnesoid X receptor agonist CDCA reduces blood pressure and regulates vascular tone in spontaneously hypertensive rats. J. Am. Soc. Hypertens. 9, 507-516.e7 (2015).

135. Tsai, C.C. et al. Cholesterol-lowering potentials of lactic acid bacteria based on bile-salt hydrolase activity and effect of potent strains on cholesterol metabolism in vitro and in vivo. ScientificWorldJournal 2014, 690752 (2014).

136. Ghosh Laskar, M., Eriksson, M., Rudling, M. \& Angelin, B. Treatment with the natural FXR agonist chenodeoxycholic acid reduces clearance of plasma LDL whilst decreasing circulating PCSK9, lipoprotein(a) and apolipoprotein C-III. J. Intern. Med. 281, 575-585 (2017).

137. Mathews, S.T. et al. Alpha2-HSG, a specific inhibitor of insulin receptor autophosphorylation, interacts with the insulin receptor. Mol. Cell. Endocrinol. 164, 87-98 (2000).

138. Galbo, T. et al. Saturated and unsaturated fat induce hepatic insulin resistance independently of TLR-4 signaling and ceramide synthesis in vivo. Proc. Natl. Acad. Sci. U.S.A. 110, 12780-12785 (2013).

139. Galbo, T. \& Shulman, G.I. Lipid-induced hepatic insulin resistance. Aging (Albany.NY) 5, 582-583 (2013).

140. Marcuccilli, M. \& Chonchol, M. NAFLD and chronic kidney disease. Int. J. Mol. Sci. 17, 562 (2016).

141. Badman, M.K. \& Flier, J.S. The adipocyte as an active participant in energy balance and metabolism. Gastroenterology 132, 2103-2115 (2007).

142. Byrne, C.D. Ectopic fat, insulin resistance and non-alcoholic fatty liver disease. Proc. Nutr. Soc. 72, 412-419 (2013).

143. Shoelson, S.E., Herrero, L. \& Naaz, A. Obesity, inflammation, and insulin resistance. Gastroenterology 132, 2169-2180 (2007).

144. Sabio, G. et al. A stress signaling pathway in adipose tissue regulates hepatic insulin resistance. Science 322, 1539-1543 (2008).

145. Tilg, H. \& Moschen, A.R. Evolution of inflammation in nonalcoholic fatty liver disease: the multiple parallel hits hypothesis. Hepatology 52, 1836-1846 (2010).

146. Adolph, T.E., Grander, C., Grabherr, F. \& Tilg, H. Adipokines and nonalcoholic fatty liver disease: multiple interactions. Int. J. Mol. Sci. 18 (2017).

147. Hotamisligil, G.S. Endoplasmic reticulum stress and the inflammatory basis of metabolic disease. Cell 140, 900-917 (2010).

148. Shoelson, S.E., Lee, J. \& Goldfine, A.B. Inflammation and insulin resistance. J. Clin. Invest. 116, 1793-801 (2006).

149. Semenkovich, C.F. Insulin resistance and atherosclerosis. J. Clin. Invest. 116, 1813-1822 (2006). 
150. Alessi, M.C. \& Juhan-Vague, I. Metabolic syndrome, haemostasis and thrombosis. Thromb. Haemost. 99, 995-1000 (2008).

151. Targher, $\mathrm{G}$ et al. Nonalcoholic fatty liver disease as a contributor to hypercoagulation and thrombophilia in the metabolic syndrome. Semin. Thromb. Hemost. 35, 277-287 (2009).

152. Targher, G \& Byrne, C.D. Diagnosis and management of nonalcoholic fatty liver disease and its hemostatic/thrombotic and vascular complications. Semin. Thromb. Hemost. 39, 214-228 (2013).

153. Sookoian, $\mathrm{S}$ et al. Circulating levels and hepatic expression of molecular mediators of atherosclerosis in nonalcoholic fatty liver disease. Atherosclerosis 209, 585-591 (2010).

154. Sookoian, $\mathrm{S}$ et al. Liver transcriptional profile of atherosclerosis-related genes in human nonalcoholic fatty liver disease. Atherosclerosis 218, 378-385 (2011).

155. Shakeri, H. et al. Consumption of synbiotic bread decreases triacylglycerol and VLDL levels while increasing HDL levels in serum from patients with type-2 diabetes. Lipids 49, 695-701 (2014).

156. Rinella, M.E. \& Sanyal, A.J. Management of NAFLD: a stage-based approach. Nat. Rev. Gastroenterol. Hepatol. 13, 196-205 (2016).

157. Vilar-Gomez, E. et al. Weight loss through lifestyle modification significantly reduces features of nonalcoholic steatohepatitis. Gastroenterology 149, 367-378 (2015).

158. Tahrani, A.A., Bailey, C.J., Del Prato, S., Barnett, A.H. Management of type 2 diabetes: new and future developments in treatment. Lancet 378, 182-197 (2011).

159. Celio, A.C. \& Pories, W.J. A history of bariatric surgery: the maturation of a medical discipline. Surg. Clin. North Am. 96, 655-667 (2016).

160. Asrih, M. \& Jornayvaz, F.R. Diets and nonalcoholic fatty liver disease: the good and the bad. Clin. Nutr. 33, 186-190 (2014).

161. Ryan, M.C. et al. The Mediterranean diet improves hepatic steatosis and insulin sensitivity in individuals with non-alcoholic fatty liver disease. J. Hepatol. 59, 138-143 (2013).

162. Ascha, M.S. et al. The incidence and risk factors of hepatocellular carcinoma in patients with nonalcoholic steatohepatitis. Hepatology 51, 19721978 (2010).

163. Kawamura, Y. et al. Effects of alcohol consumption on hepatocarcinogenesis in japanese patients with fatty liver disease. Clin. Gastroenterol. Hepatol. 14, 597-605 (2016).

164. Marrero, J.A. et al. Alcohol, tobacco and obesity are synergistic risk factors for hepatocellular carcinoma. J. Hepatol. 42, 218-224 (2005).

165. Bacchi, E. et al. Both resistance training and aerobic training reduce hepatic fat content in type 2 diabetic subjects with nonalcoholic fatty liver disease (the RAED2 randomized trial). Hepatology 58, 1287-1295 (2013).

166. Hallsworth, K. et al. Resistance exercise reduces liver fat and its mediators in non-alcoholic fatty liver disease independent of weight loss. Gut 60, 1278-1283 (2011).

167. Oh, S. et al. Moderate to vigorous physical activity volume is an important factor for managing nonalcoholic fatty liver disease: a retrospective study. Hepatology 61, 1205-1215 (2015). 
168. Lonardo, A., Ballestri, S., Targher, G. \& Loria, P. Diagnosis and management of cardiovascular risk in nonalcoholic fatty liver disease. Expert Rev. Gastroenterol. Hepatol. 9, 629-650 (2015).

169. Ratziu, V., Goodman, Z. \& Sanyal, A. Current efforts and trends in the treatment of NASH. J. Hepatol. 62 (1 suppl), S65-S75 (2015).

170. Lombardi, R. et al. Pharmacological interventions for non-alcohol related fatty liver disease (NAFLD): an attempted network meta-analysis. Cochrane Database Syst. Rev. 3, CD011640 (2017).

171. Bugianesi, E. et al. A randomized controlled trial of metformin versus vitamin $\mathrm{E}$ or prescriptive diet in nonalcoholic fatty liver disease. Am. J. Gastroenterol. 100, 1082-1090 (2005).

172. Belfort, R. et al. A placebo-controlled trial of pioglitazone in subjects with nonalcoholic steatohepatitis. N. Engl. J. Med. 355, 2297-2307 (2006).

173. Ratziu, V. et al.; LIDO Study Group. Rosiglitazone for nonalcoholic steatohepatitis: one-year results of the randomized placebo-controlled Fatty Liver Improvement with Rosiglitazone Therapy (FLIRT) Trial. Gastroenterology 135, 100-110 (2008).

174. Haukeland, J.W. et al. Metformin in patients with non-alcoholic fatty liver disease: a randomized, controlled trial. Scand. J. Gastroenterol. 44, 853-860 (2009).

175. Ratziu, V. et al.; LIDO Study Group. Long-term efficacy of rosiglitazone in nonalcoholic steatohepatitis: results of the fatty liver improvement by rosiglitazone therapy (FLIRT 2) extension trial. Hepatology 51, 445-453 (2010).

176. Argo C.K. et al. Effects of $n-3$ fish oil on metabolic and histological parameters in NASH: a double-blind, randomized, placebo-controlled trial. J. Hepatol. 62, 190-197 (2015).

177. Armstrong M.J. et al. Liraglutide safety and efficacy in patients with nonalcoholic steatohepatitis (LEAN): a multicentre, double-blind, randomised, placebo-controlled phase 2 study. Lancet 387, 679-690 (2016).

178. Ratziu, V. et al.; GOLDEN-505 Investigator Study Group. Elafibranor, an agonist of the peroxisome proliferator-activated receptor- $\alpha$ and $-\delta$, induces resolution of nonalcoholic steatohepatitis without fibrosis worsening. Gastroenterology 150, 1147-1159 (2016).

179. Cusi, K. et al. Long-term pioglitazone treatment for patients with nonalcoholic steatohepatitis and prediabetes or type 2 diabetes mellitus: a randomized trial. Ann. Intern. Med. 165, 305-315 (2016).

180. Joy, T.R. et al. Sitagliptin in patients with non-alcoholic steatohepatitis: a randomized, placebo-controlled trial. World J. Gastroenterol. 23, 141-150 (2017).

181. Bril, F. et al. Liver safety of statins in prediabetes or T2DM and nonalcoholic steatohepatitis: post-hoc analysis of a randomized trial. J. Clin. Endocrinol. Metab. 2017 Jun 1. doi: 10.1210/jc.2017-00867 [Epub ahead of print].

182. Lonardo, A. \& Loria, P. Potential for statins in the chemoprevention and management of hepatocellular carcinoma. J. Gastroenterol. Hepatol. 27, 16541664 (2012).

183. Nascimbeni, F. et al.; LIDO study Group. Statins, antidiabetic medications and liver histology in patients with diabetes with non-alcoholic fatty liver disease. B.M.J. Open Gastroenterol. 3, e000075 (2016).

184. Athyros, V.G. et al. The use of statins alone, or in combination with pioglitazone and other drugs, for the treatment of non-alcoholic fatty liver 
disease/non-alcoholic steatohepatitis and related cardiovascular risk. An Expert Panel statement. Metabolism 71, 17-32 (2017).

185. Glen, J., Floros, L., Day, C. \& Pryke, R.; Guideline Development Group. Non-alcoholic fatty liver disease (NAFLD): summary of NICE guidance. B.M.J. 354, i4428 (2016). 


\section{FIGURES LEGENDS}

Figure 1. Proposed pragmatic algorithm for the management of suspected NAFLD in patients with established diabetes mellitus.

The algorithm has been developed using both available evidence and guidelines, as well as expert opinion where uncertainty exists and evidence was not available. Patients with type 1 or type 2 diabetes should routinely undergo diagnostic procedures for the diagnosis of NAFLD, which relies on the demonstration of hepatic steatosis. Serum transaminase levels are not reliable indicators for the screening and diagnosis of NAFLD and should not be used without further investigation in clinical practice. Liver ultrasonography is the preferred first-line imaging method for the diagnosis of NAFLD. The exclusion of competing causes of hepatic steatosis, e.g., excessive alcohol intake, viral hepatitis, autoimmune hepatitis, hemochromatosis or chronic use of steatogenic medications is key for the diagnosis of NAFLD. The algorithm can be used to select those NAFLD patients to submit to liver biopsy, or if biopsy is not undertaken, the noninvasive assessment of advanced liver fibrosis according to panels of specific serum biomarkers (e.g., the fibrosis-4 [FIB-4] score, the NAFLD fibrosis score or the enhanced liver fibrosis [ELF] blood test) and transient elastography (e.g., the FibroScan or other non-invasive imaging techniques that assess liver stiffness) may be used for selecting patients for upper gastrointestinal endoscopy (aimed at showing the presence of esophageal/gastric varices due to portal hypertension) and long-term surveillance for liver-related complications, including hepatocellular carcinoma, always if cirrhosis is present; and in selected non-cirrhotic cases, particularly if advanced fibrosis is present).

Figure 2. Prevalence of clinically manifest cardiovascular disease (CVD) in patients with type 2 diabetes mellitus.

Sex- and age-adjusted prevalence of coronary (defined as myocardial infarction, angina pectoris or coronary revascularization procedures), cerebrovascular (ischemic stroke, recurrent transient ischemic attacks, carotid endarterectomy or carotid stenosis $>70 \%$ as diagnosed by echo-Doppler scanning) and peripheral (intermittent claudication, rest pain, as confirmed by echo-Doppler scanning, lower extremity amputations or peripheral revascularization procedures) in type 2 diabetic outpatients with (red columns) and without (green columns) ultrasound-diagnosed NAFLD. Data derived from Targher et al. ${ }^{28}$ (Reproduced with permission). 
Figure 3. Prevalence of diabetic nephropathy and retinopathy in patients with type 2 diabetes mellitus.

Age- and sex-adjusted prevalence of chronic kidney disease (CKD defined as estimated glomerular filtration rate $[\mathrm{eGFR}]<60 \mathrm{ml} / \mathrm{min} / 1.73 \mathrm{~m}^{2}$ or overt proteinuria), and diabetic retinopathy in type 2 diabetic outpatients with (red columns) and without (green columns) ultrasound-diagnosed NAFLD. Data derived from Targher et al. ${ }^{90}$ (Reproduced with permission).

Figure 4. Potential pathways, factors and processes that link dysbiosis, gut microbial mediators and alterations in hepatic structure and function (NAFLD) to cardiovascular risk factors and vascular and renal diseases.

Dysbiosis is associated with increased intestinal permeability and intestinal dysfunction (A). Factors in $(A)$ increase risk of NAFLD via alterations in several pathways, factors and molecules that modify liver structure and function in NAFLD (B). With these liverspecific changes (B), there is an increase in risk factors for vascular disease $(C)$ and the subsequent development over time of vascular and renal diseases via increased insulin resistance, metabolic syndrome and structural and functional changes affecting both the vasculature and the kidneys (D).

Figure 5. Potential pathways linking dysbiosis to cardiovascular and chronic kidney disease.

Intestinal dysbiosis perturbs bile acid metabolism affecting the levels of e.g. bile acids (such as chenodeoxycholic acid [CDCA]), short-chain fatty acids (SCFA), trimethylamine and lipopolysaccharide. Via decreased activity of hepatic nuclear receptor farnesoid $X$ receptor (FXR) activity in NAFLD and increased intestinal permeability, there is a further increase in cardio-renal risk factors with subsequent development of vascular and renal diseases.

Figure abbreviations: LDL-C, low-density lipoprotein cholesterol; PCSK9, proprotein convertase subtilisin/kexin type 9; Cyp7a1, cytochrome p450 7a1; Lp(a), lipoprotein (a); apoCIII, apolipoprotein CIII; TGRLPs, triglyceride rich lipoproteins; TMAO, trimethylamine oxide.

Figure 6. Liver-specific pathways in NAFLD linking NAFLD to cardiovascular and chronic kidney disease. 
A variety of factors, such as visceral adipose tissue accumulation, low-grade chronic inflammation, T2DM, dysbiosis and dietary factors, can affect liver-specific pathways to induce (or amplify) lipotoxicity, insulin resistance, oxidative stress and chronic inflammation. Consequent Kupffer cell and stellate cell activation may occur and the disease process progresses, with further inflammation, collagen deposition and fibrosis into the liver. All of these intrahepatic processes may also increase the production of risk factors for vascular disease with subsequent development of both cardiovascular disease and chronic kidney disease.

Figure abbreviations: DAGs, di-acylglycerols; di-P PA, di-palmitoyl phosphatidic acid; LCFAs, long chain fatty; LDL-C, low-density lipoprotein cholesterol; TAGs, tri-acyl glycerols; PNPLA3, patatin-like phospholipase domain containing 3; TM6SF2, transmembrane 6 superfamily member 2; TGF-beta, transforming growth factor-beta; TNF-alpha, tumor necrosis factor. 